\title{
Robert Schöllhorn: a tribute on the occasion of his 75th birthday
}

\author{
Alexander Nemudry • Werner Paulus • Hellmut Eckert
}

Published online: 3 February 2011

(C) Springer-Verlag 2011

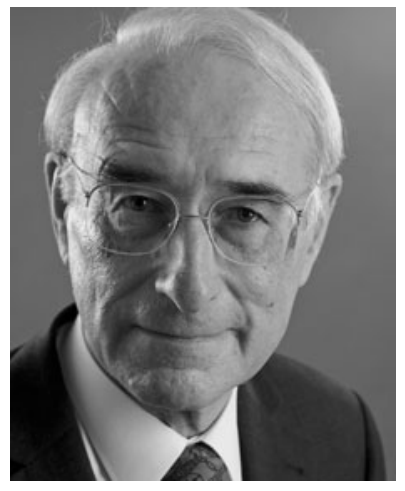

As a commitment, as well as appreciation, and in celebration of the 75th birthday of Professor Robert Schöllhorn, this special issue is composed of contributions from his former colleagues and students. It is also a tribute

A. Nemudry $(\bowtie)$

Institute of Solid State Chemistry and Mechanochemistry SB RAS, Novosibirsk, Russia

e-mail: nemudry@solid.nsc.ru

\section{H. Eckert}

Institut für Physikalische Chemie,

Westfälische Wilhelms-Universität Münster,

Corrensstr. 28/30,

48149 Münster, Germany

e-mail: eckerth@uni-muenster.de

\section{W. Paulus}

Sciences Chimiques de Rennes-UMR 6226,

Université de Rennes 1,

Matériaux Inorganiques: Chimie Douce et Réactivité,

Campus de Beaulieu, Bât 10B,

35042 Rennes, France

e-mail: werner.paulus@univ-rennes1.fr

in respect for his integrity, curiosity, and enthusiasm having influenced many colleagues, students, and science through his pioneering work in the field of solid state chemistry and its reactivity.

Although in early days of his outstanding scientific career, Robert Schöllhorn has been interested in the understanding of the reactivity of molecular systems such as the reduction and fixation of molecular nitrogen, his scientific identity and reputation is since more than four decades connected to the field of intercalation chemistry and the understanding of solid state reaction mechanisms.

He explored a large variety of guest-host systems and their respective interactions with the vision of reaching a better understanding of the underlying reaction mechanisms and related physical properties. Always pushing to utilize equipment at the forefront of instrumental development, his pioneering work provided unique insights into the kinetics, mechanisms, and the structural and dynamic aspects of solid-state reactions and intercalation chemistry. Particular highlights of this work include the in situ study of reacting systems by real time neutron diffraction (shortly after the installation of the high-flux neutron reactor at the ILL Grenoble) and the application of solid-state nuclear magnetic resonance techniques (together with his long-term collaborator Werner Müller-Warmuth) to localize the intercalated guest molecules and to characterize their dynamics within these highly anisotropic environments. Based on the systematic analysis and exploration of a large variety of systems with different dimensionalities, he recognized the intercalation process of neutral molecules into metallic and semiconducting host lattices as a topotactical redox reaction with simultaneous electron/ ion transfer. This conceptual breakthrough, summarized in the article "Reversible Topotactic Redox Reactions of 
Solids by Electron-Ion Transfer ", (Angewandte Chemie, 1980) remains one of his most influential contributions to this research area. It opened the door to the controlled preparation of non-stoichiometric compounds via coulometric synthesis and was quickly adopted by many laboratories all over the world. Many of the new electroactive materials, which are nowadays being used as electrode components in mobile energy sources, such as lithium ion batteries and fuel cells, originate from this central idea. From today's perspective, we can state that Robert Schöllhorn's work has laid the foundations and contributed significantly to the enormous progress witnessed in this research area.

In appreciation of his excellent science and of the great scientific impact his insights and ideas have made in developing the field of solid-state electrochemistry to where it stands today, we dedicate this special volume to Robert Schöllhorn in honor of his 75th birthday. 